\title{
O teste KTK na avaliação da coordenação motora de crianças e suas relações com
}

\section{antropometria e desempenho motor: revisão sistemática}

\author{
The KTK test in the assessment of children's motor coordination and its relationship with \\ anthropometry and motor performance: a systematic review \\ EI test KTK en la evaluación de la coordinación motora infantil y su relación con la antropometría \\ y el desempeño motor: una revisión sistemática
}

Recebido: 20/01/2022 | Revisado: 29/01/2022 | Aceito: 04/02/2022 | Publicado: 06/02/2022

José Irineu Gorla

ORCID: https://orcid.org/0000-0003-2099-9643 Universidade Estadual de Campinas, Brasil E-mail: jigorla@uol.com.br

Karina Santos Guedes de Sá

ORCID: https://orcid.org/0000-0001-7027-526X Universidade Estadual de Campinas, Brasil E-mail: karina-sa@outlook.com

Ygor Carrozzini Macedo de Mattos

ORCID: https://orcid.org/0000-0003-0465-7432

Universidade Estadual de Campinas, Brasil

E-mail: ycarrozzini@gmail.com

Flávio Henrique Corrêa

ORCID: https://orcid.org/0000-0001-6124-6539

Universidade Estadual de Campinas, Brasil

E-mail: flavio.correa76@gmail.com

Nayara Christine Souza

ORCID: https://orcid.org/0000-0002-1032-6034

Universidade Estadual de Campinas, Brasil

E-mail: naysouzac@gmail.com

Jéssica Reis Buratti

ORCID: https://orcid.org/0000-0001-9953-7817

Universidade Estadual de Campinas, Brasil

E-mail: jerburatti@gmail.com

Matheus Jancy Bezerra Dantas

ORCID: https://orcid.org/0000-0003-4743-0510

Universidade Estadual de Campinas, Brasil

E-mail: matheusjancy@gmail.com

Thaísa Lucas Filgueira Souza Dantas

ORCID: https://orcid.org/0000-0002-9976-3205

Universidade Estadual de Campinas, Brasil

E-mail: thaisasdantas@gmail.com

Anselmo De Athayde Costa e Silva

ORCID: https://orcid.org/0000-0001-5265-619X

Universidade Federal do Pará, Brasil

E-mail: anselmocs@ufpa.br

\begin{abstract}
Resumo
Objetivo: Identificar o que tem sido publicado a respeito da utilização do teste KTK nos últimos anos para avaliação da coordenação motora de crianças, e secundariamente analisar a relação entre coordenação motora, antropometria e desempenho. Métodos: Foi realizada uma revisão sistemática nas bases de dados do PubMed e Scielo, através da estratégia de busca (test KTK) AND (motor coordination). Caracterizavam-se como critérios de inclusão: artigos em língua inglesa ou em português; artigos de estudos observacionais ou de intervenção e pesquisas que foram realizadas entre os anos 2015 - 2021. Resultados: Um total de 11.221 crianças compõem a amostra total dos estudos. Medidas antropométricas apresentam uma relação inversamente proporcional com a coordenação motora, enquanto o desempenho apresenta uma relação diretamente proporcional. O perfil populacional demonstra que alguns fatores antropométricos, idade avançada, prática esportiva e características de temperamento específicas de atividade e atenção-persistência, foram os preditores mais fortes para uma melhor coordenação motora. Conclusão: A literatura
\end{abstract}


recente demonstra que esse teste possui relações inversamente proporcionais e diretamente proporcional com medidas antropométricas e o desempenho, respectivamente.

Palavras-chave: Avaliação; Coordenação motora grossa; Körperkoordinations Test für Kinder.

\begin{abstract}
Objective: To identify what has been published regarding the use of the KTK test in recent years to assess children's motor coordination, and secondarily analyze the relationship between motor coordination, anthropometry, and performance. Methods: A systematic review was carried out in the PubMed and Scielo databases, using the search strategy (test KTK) AND (motor coordination). Inclusion criteria were articles in English or Portuguese; articles from observational or intervention studies and surveys that were carried out between the years 2015 - 2021. Results: A total of 11,221 children make up the total sample of studies. Anthropometric measurements show an inversely proportional relationship with motor coordination, while performance shows a directly proportional relationship. The population profile demonstrates that some anthropometric factors, advanced age, sports practice and specific temperament characteristics of activity and attention-persistence were the strongest predictors for better motor coordination. Conclusion: Recent literature demonstrates that this test has inversely and directly proportional relationships with anthropometric measurements and performance, respectively.
\end{abstract}

Keywords: Evaluation; Gross motor coordination; Körperkoordinations Test für Kinder.

\title{
Resumen
}

Objetivo: Identificar lo que se ha publicado sobre el uso de la prueba KTK en los últimos años para evaluar la coordinación motora de los niños, y secundariamente analizar la relación entre la coordinación motora, la antropometría y el rendimiento. Métodos: Se realizó una revisión sistemática en las bases de datos PubMed y Scielo, utilizando la estrategia de búsqueda (test KTK) AND (coordinación motora). Los criterios de inclusión fueron: artículos en inglés o portugués; artículos de estudios observacionales o de intervención y encuestas que se realizaron entre los años 2015 - 2021. Resultados: Un total de 11.221 niños conforman la muestra total de estudios. Las medidas antropométricas muestran una relación inversamente proporcional con la coordinación motora, mientras que el rendimiento muestra una relación directamente proporcional. El perfil de la población demuestra que algunos factores antropométricos, la edad avanzada, la práctica deportiva y las características específicas del temperamento de la actividad y la atención-persistencia fueron los predictores más fuertes para una mejor coordinación motora. Conclusión: La literatura reciente demuestra que esta prueba tiene relaciones inversa y directamente proporcionales con las medidas antropométricas y el rendimiento, respectivamente.

Palabras clave: Evaluación; Coordinación motora gruesa; Körperkoordinations Test für Kinder.

\section{Introdução}

A coordenação motora é a capacidade de realizar movimentos de forma harmoniosa e coordenada, envolvendo os sistemas nervoso, muscular, esquelético e sensorial (Bernshtein, 1967). A coordenação motora é dividida em fina e grossa. A coordenação motora fina compreende a realização de movimentos mais precisos que utilizam músculos pequenos, como os movimentos de pinça da mão. Já a coordenação motora grossa, temos o envolvimento de grandes músculos para a realização de movimentos maiores como correr, pular, subir e descer escadas, entre outros (A. Santos, 2002).

A experiência motora permite que os componentes da motricidade se desenvolvam de forma ampla, o que possibilita a execução dos movimentos simples e complexos de maneira equilibrada e eficiente (Buratti et al., 2020). Avaliar a coordenação motora de crianças, permite identificar precocemente atrasos no desenvolvimento psicomotor, o que permite que sejam realizadas intervenções precocemente que evitarão maiores complicações futuramente. Diversos testes para a análise do padrão de desempenho motor em crianças e adolescentes são apresentados na literatura científica. Um dos testes mais utilizados para avaliar coordenação motora grossa de crianças é o teste Körperkoordinationstest Für Kinder (KTK).

O teste KTK é um instrumento composto por diferentes tarefas de estabilidade do equilíbrio dinâmico, energia dinâmica dos membros inferiores, velocidade, ritmo e fluidez de movimento (Gorla et al., 2009) e poderia ajudar os pesquisadores a compreender o desempenho motor de crianças. A duração aproximada do teste é de 20 minutos, sendo prático e oferecendo uma avaliação completa do desempenho motor grosso da criança. O teste é composto de quatro tarefas: tarefa 1: trave de equilíbrio; tarefa 2: saltos monopedais; tarefa 3: saltos laterais e tarefa 4: transferências laterais.

A coordenação motora está favoravelmente associada a marcadores de saúde, como atividade física, condicionamento 
cardiorrespiratório, força muscular, resistência e estado de peso saudável durante toda a infância. As duas principais variáveis que influenciam o desempenho motor no teste KTK são fatores antropométricos e a prática de atividades físicas (Nascimento et al., 2019)

Visto que o teste KTK é um dos testes mais utilizados para avaliar coordenação motora de crianças, e sabendo que não existe na literatura uma revisão focada em analisar a relação entre coordenação motora e as duas principais variáveis que influenciam o desempenho motor no teste KTK, os objetivos desta revisão sistemática são identificar o que tem sido publicado a respeito da utilização do teste KTK nos últimos anos para avaliação da coordenação motora de crianças, e secundariamente analisar a relação entre coordenação motora, antropometria e desempenho.

\section{Metodologia}

Este estudo é caracterizado como uma revisão sistemática e segue as diretrizes da declaração de itens de relatório preferidos para revisões sistemáticas e meta-análise (PRISMA) (Moher et al., 2009). Dessa maneira, é uma investigação científica realizada para avaliar e conduzir uma síntese dos resultados de múltiplos estudos primários.

Para a realização dessa revisão foram utilizadas as bases de dados do PubMed e Scielo, através da estratégia de busca (test KTK) AND (motor coordination). As buscas foram realizadas entre os meses de outubro e novembro de 2021. Inicialmente os artigos foram selecionados através da leitura do título e resumo, aqueles com potencial para responder ao objetivo desta revisão foram lidos na íntegra e os critérios de inclusão e exclusão aplicados.

Caracterizavam-se como critérios de inclusão: artigos em língua inglesa ou em português; artigos de estudos observacionais ou de intervenção e pesquisas que foram realizadas entre os anos 2015 - 2021. Foi considerado como critério de exclusão artigos de revisão e resumos em anais de congressos (Figura 1). 
Figura 1. Diagrama de fluxo PRISMA.
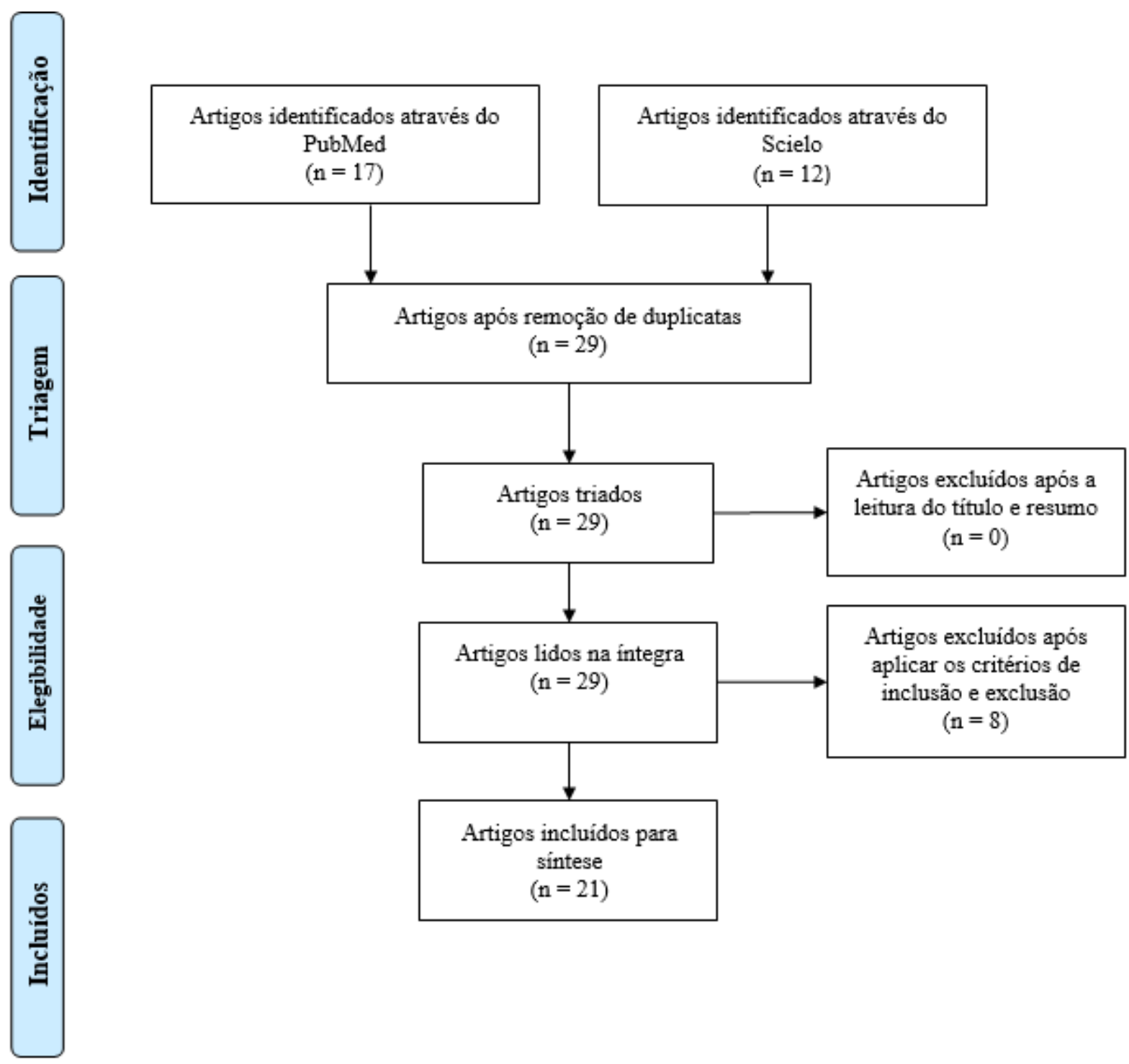

Fonte: Autores, adaptado de http://www.prisma-statement.org/PRISMAStatement/FlowDiagram

\section{Resultados}

Através das buscas sistemáticas nas bases de dados, foram encontrados 29 artigos (PubMed = 17; Scielo = 12). Não foram identificadas duplicatas entre as bases de dados. Foram excluídos sete artigos por não atenderem aos critérios de inclusão (revisão sistemática $\mathrm{N}=1$; artigos anteriores a $2015 \mathrm{~N}=6$ ) e um artigo foi excluído pois não possuía acesso livre, o arquivo foi solicitado junto aos autores, porém não houve resposta. Desta forma, foram incluídos nesta revisão 21 artigos.

Um total de 11.221 crianças (5.631 meninos e 5.166 meninas) participaram dos estudos (um artigo não especificou o número de meninos e meninas). O mapa de publicações demonstra uma grande abrangência, possuindo representantes em quase todos os continentes: No Brasil foram encontrados 8 artigos, em Portugal 5 artigos, na Finlândia 2 e Turquia, Moçambique, Austrália, Peru, México e Sérvia com uma publicação cada. Com relação aos objetivos dos estudos, é possível dividir em três grandes grupos: 1) Que relacionaram a coordenação motora com características antropométricas, 2) Que relacionaram a coordenação motora com aspectos do desempenho e 3) Que traçaram perfis de populações. Os resumos dos artigos presentes nesta revisão estão dispostos na Tabela 1. 


\subsection{Coordenação motora e antropometria}

Um total de sete artigos avaliaram a relação entre a coordenação motora e medidas antropométricas (Freitas et al., 2017; Lima et al., 2017; Lopes et al., 2015, 2018; Lopes; Utesch; Rodrigues, 2020; Luz et al., 2016; Tchamo et al., 2017) e dois artigos não tinham como objetivo principal, porém trouxeram resultados sobre essa relação (Henrique et al., 2018; Valdívia et al., 2018).

Os resultados demonstraram que o índice de massa corporal, a circunferência da cintura, o percentual de gordura corporal, a razão cintura/estatura, estatura e massa gorda são inversamente proporcionais à coordenação motora em ambos os sexos, ou seja, quanto maiores forem essas medidas antropométricas, menores são os resultados de coordenação motora (Freitas et al., 2017; Lopes et al., 2018; Lopes; Utesch; Rodrigues, 2020; Luz et al., 2016). Além disso, crianças que nascem com baixo peso podem apresentar alterações na força muscular e coordenação motora (Tchamo et al., 2017). Estima-se que crianças com IMC e peso mais altos tiveram 5,44 e 5,15 vezes mais chance de apresentar coordenação motora inferior (Lima et al., 2017). Apenas um estudo apresentou uma relação antropometria X coordenação motora positiva (Lopes et al., 2015).

\subsection{Coordenação motora e desempenho}

Dez artigos relacionaram a coordenação motora com o desempenho em atividades físicas e esportes (Almeida et al., 2021; Barros et al., 2020; Laukkanen et al., 2017; Lopes et al., 2017; Luz et al., 2018; NOBRE et al., 2017; Ochoa-Martínez et al., 2019; Popović et al., 2020; Rudd et al., 2017; Söğüt, 2016).

No geral, os resultados mostram uma relação diretamente proporcional entre o desempenho e a coordenação motora grossa, ou seja, quanto melhor forem os resultados em testes físicos de desempenho, melhor são os resultados da coordenação motora grossa (Luz et al., 2018). Além disso, crianças que praticam atividades físicas regulares ou esportes apresentam melhores resultados de coordenação motora grossa quando comparados com aqueles que não praticam, inclusive de crianças com deficiência auditiva e síndrome de Down (Almeida et al., 2021; Barros et al., 2020; Laukkanen et al., 2017; Lopes et al., 2017; Nobre et al., 2017; Ochoa-Martínez et al., 2019; Söğüt, 2016). Ademais, aquelas que praticam atividades poliesportivas apresentam melhores resultados de coordenação quando comparados com crianças que praticam apenas uma modalidade (Popović et al., 2020). Um dos estudos não encontrou resultados que demonstrem que o treinamento da ginástica afeta a coordenação motora grossa (Rudd et al., 2017).

\subsection{Perfis populacionais}

Quatro artigos se propuseram a traçar perfis populacionais e descrever cálculos para escore fatorial do teste KTK (Henrique et al., 2018; Moreira et al., 2019; Niemistö et al., 2020; Valdívia et al., 2018).

Ao se comparar a coordenação motora entre irmãos, identificou-se que no geral os pares irmão-irmão e os irmãos mais velhos apresentaram maiores escores de coordenação motora, entretanto os pares irmã-irmã apresentaram maior semelhança na coordenação motora grossa (Valdívia et al., 2018). Além disso, menor peso, menor índice de massa corporal e gordura subcutânea, idade avançada, participação mais frequente em esportes e características de temperamento específicas de atividade e atenção-persistência, foram os preditores mais fortes para uma melhor coordenação motora (Henrique et al., 2018; Niemistö et al., 2020). 
Tabela 1. Descrição dos artigos.

ARTIGO OBJETIVO

AMOSTRA PROTOCOLO

Determinar a capacidade de diferentes medidas de adiposidade L. Lopes et al. para diferenciar pouca/ampla CM; e 2015) avaliar a relação entre diferentes avaliar a relação entre diferentes
medidas de adiposidade e medidas de adipo

Medidas antopométricas foran medidas (peso, menianças $(378$ meninas) Itura e circunferência da cintura, percentual gordura, índice de massa corporal (IMC) e relação cintura-altura). A coordenação motora foi avaliada pelo teste KTK. A aptidão cardiorrespiratória fo predita por um teste de corrida vaivém de $20 \mathrm{~m}$.

101 tenistas juniores $(50$ junior $(50$ meninas)

(Söğüt, 2016) Deteminar os níveis desempenho da coordenação motora grossa de tenistas juniores e analisar os efeitos da idade e do gênero.

Analisar a associação do estado maturacional com o desempenho nas provas de coordenação pentor

L. G. O. Luz et nas provas

al., 2016) em crianças e examinar se a relação entre o estado desempenho no KTK é mediada por alguma medida antropométrica.

Analisar o rastreamento e

1744 crianças $(875$ meninos e 798 meninas)
(Lima et al., 2017) estabilidade da coordenação motora

(Lima et al., 2017) estabilidade da coordenação motora
em crianças de 6 anos a 9 e 13 anos.

\begin{tabular}{|c|c|c|}
\hline $\begin{array}{l}\text { (L. Lopes et al., } \\
\text { 2017) }\end{array}$ & $\begin{array}{l}\text { Examinar a relação entre } \\
\text { flexibilidade e MC em crianças }\end{array}$ & $\begin{array}{c}596 \text { crianças ( } 315 \\
\text { meninos e } 281 \\
\text { meninas) }\end{array}$ \\
\hline $\begin{array}{c}\text { (Nobre et al., } \\
\text { 2017) }\end{array}$ & $\begin{array}{l}\text { Analisar os efeitos de um protocolo } \\
\text { de treinamento pliométrico sobre a } \\
\text { composição corporal e desempenho } \\
\text { motor de meninos com } \\
\text { sobrepeso/obesidade de } 7 \text { a } 9 \text { anos }\end{array}$ & $\begin{array}{l}59 \text { meninos (grupo } \\
\text { treinamento } \\
\text { pliométrico }(\mathrm{T}= \\
40) \text { e grupo } \\
\text { controle }(\mathrm{C}=19))\end{array}$ \\
\hline
\end{tabular}

Utilizou o teste KTK para avaliar a coordenação motora grossa (GMC)

Os dados antropométricos foram medidos (altura, massa corporal, altura sentada, circunferência da cintura, índice de massa corporal (IMC), massa gorda e massa livre de gordura). A maturação go biologica for avaliada pela porcentagen da estatura pelo teste KTK.

A coordenação motora foi avaliada através do teste KTK. A pontuação de desempenho bruto fo convertida em uma "pontuação" padronizada MQScore total E foram classificados como baixo (MQScore $<85)$, normal $(\mathrm{MQ}$-Score $=85-115)$ ou alto (MQ-Score> 115).

A coordenação motora foi avaliada pelo teste KTK. A aptidão cardiorrespiratória foi avaliada pelo teste A $\mathrm{m}$ shuttle run, a forç muscular pelos testes curtA up e push up. A flexibilidade foi avaliada pelo teste
de sentar e alcançar e levantar o tronco.

A coordenação motora grossa foi avaliada pelo teste KTK. A aptidão Tisica foi avaliada pela força $d$ preensão manual, salto em distância em pé (SLJ), curlups, sentar e alcançar, teste do quadrado, velocidade de corrida e teste de corrida em milhas. Foi aplicado nos participantes treinamento pliométricos para membros inferiores $(20 \mathrm{~min} / \mathrm{di}$ duas vezes por semana, durante 12 semanas).

\section{PRINCIPAIS RESULTADOS}

As análises de regressão logística demonstraram que o índice de massa corporal ( $\beta=2,155$; Intervalo de Confiança (IC) $95 \%$ : 1,164-3,992; $\mathrm{p}=0,015$ para meninas; $\beta=3,255$; IC 95\%: 1,740-6,088; $p<0,001$ para meninos), a circunferência da cintura ( $\beta=2,489$; IC 95\%: $1,242-4,988 ; p=0,010$ para meninas; $\beta=3,296$; IC 95\%: 1,784-6,090; $p<0,001$ para meninos), o percentual de gordura corporal ( $\beta=2,395$; IC 95\%: $1,234-4,646 ; p=0,010$ para meninas; $\beta=2,603$; IC 95\%: $1,462-4,634 ; p<0,001$ para m; $p=0,010$ razão meninos) estavam posiva e sigiflcativat $2,025-7,283$; $\mathrm{p}<0.001$ pa meninos) estavam positiva e significativamente relacionados à coordenação motora en ambos os sin $(\beta=1,343$; IC 95\%: 0,713-2,528; $p=0,381)$

Não houve diferença significativa de gênero e idade nos subtestes do KTK e no quociente motor total. $40,6 \%$ dos participantes tiveram desempenho acima do normal, o que sugere que crianças que praticam esportes podem apresentar melhores valores de GMC.

Estatura, massa corporal, perímetro de cintura e massa gorda apresentaram correlação inversa de magnitude pequena a moderada com as provas de coordenação motora. A maturação biológica associou-se significativamente prova de equilíbrio em marcha à retaguarda $(\mathrm{r}=-0,34)$. Foi identificada mediação total do perímetro de cintura na relação do estado maturacional com o equilíbrio em marcha à retaguarda (77\%).

Os coeficientes de rastreamento entre o escore MQ e cada elemento KTK em diferentes idades foram moderados ( $r>0,35$ ). Crianças com IMC e peso mais altos tiveram 5,44 e 5,15 vezes mais chance de apresentar CM inferior.

Os participantes dos grupos da zona saudável de ambos os testes de flexibilidade exibiram escores significativamente melhores de MC em comparação com participantes com resultados abaixo da zona saudável ( $\mathrm{P}$ $<0,001)$.

$\mathrm{O}$ exercício pliométrico melhorou a força de preensão manual $(\mathrm{d}=0,23)$ sentar e alcançar $(d=0,18)$, flexões $(d=0,39)$, salto em distância em pé $(d=$ $0,80)$, agilidade $(d=0,48)$ e tempo no teste de milha corrida $(d=0,38)$. Para resultados de coordenação motora grossa, o grupo $\mathrm{T}$ apresentou melho desempenho em todos os testes após o treinamento pliométrico com tamanho de efeito moderado / grande. 
Avaliar associações entre baixo peso ao nascer (BPN) peso ao nasce

(Tchamo et al., antropont corporal, aptidão física e escolares de Maputo, Moçambique.

353 crianças (Não especificou entre meninos e meninas) (composição corporal, índices de peso para idade, altura para idade e peso para estatura). A aptidão física foi avaliada pela força de preensão manual, flexibilidade, agilidade, salto em distância velocidade de corrida. A coordenação motora grossa foi avaliada através do teste KTK.

Investigar associações entre o IMC

(Laukkanen et al., na primeira infância e o IMC, motora $(\mathrm{MC})$ na meia-infância.

64 crianças

coordenacão motora foi avaliada pelo teste KTK 32 foi avaliada usando acelerômetros triaxiais.

Avaliar a eficácia de um programa de ginástica de 16 semanas na estabilidade, habilidades de controle de objetos
coordenação geral do corpo.

(Rudd et al.,

As crianças do grupo intervenção (16 semanas x 2 horas de ginástica) foram comparadas às criança 170 que receberam ( $16 \times 2$ horas $)$ o currículo d meninas) educação física padrão. A coordenação moto rossa foi avaliada através do teste KTK.

(Freitas et al., $\begin{aligned} & \text { Verificar a relação entre o índice de } \\ & \text { massa corporal (IMC) e }\end{aligned}$ 2017) coordenação motora de jovens atletas de atletismo.

(V. P. Lopes et Avaliar a relação entre o IMC e

coordenação motora (CM) em crianças de 6 a 10 anos.

4 meninos

Foram avaliadas medidas antropométricas (índice de massa corporal (IMC)) e a coordenação motor foi avaliada através do teste KTK.

$3.738(1.912 \quad$ Medidas antropométricas foram avaliadas (índice de meninos e 1.826 massa corporal (IMC)), A coordenação motor grossa foi avaliada através do teste KTK. coordenação motora (bateria KTK).

Avaliar as relações multivariadas coordenação motora

74 meninas

Investigar a semelhança entre irmãos na coordenaç̃̃ mo gross (GMC), bem como 2018)
correlatos biológicos e ambientai
do GMC entre crianças peruanas. 2018)
correlatos biológicos e ambienta
do GMC entre crianças peruanas.

1.256 irmãos biológicos $(560$

meninos e 696

meninas

GMC foi avaliado através do teste KTK. Dado Investigar $\mathrm{o}$ rastreamento da coordenação motora grossa (GMC) e traçar o perfil de crianças aos 6 $\begin{array}{cl}\text { Henrique et al., } & \text { e traçar o perfil de crianças aos } 6 \\ \text { 2018) } & \text { anos de idade que apresentaram }\end{array}$ padrões de estabilidade mais GMC.

(Moreira et al., Investigar a estrutura fatorial de
245 crianças $(123$

meninos e 122

meninas)

565 voluntários

(282 meninos e 283 meninas)
C) física $(\mathrm{PF})$ também foram medidos.
Foram avaliadas antropometria, atividade física aptidão física. A coordenação motora foi avaliad através do teste KTK. KTK em uma amostra brasileira; e,
comparar quatro possibilidades de

Foram avaliadas dimensões corporais, oito itens de condicionamento físico e quatro tarefas de

As crianças com baixo peso ao nascer eram mais leves e menores do que as crianças com peso normal ao nascer. Eles também mostraram um desempenho reduzido em testes de força de preensão manual e movimentos laterais. Essas diferenças permaneceram significativas mesmo após o ajuste para idade, sexo, tamanho corporal e espessura da dobra cutânea de gordura. Esses resultados sugerem que o baixo peso ao nascer podem influenciar na força muscular e coordenação motora de crianças.

\section{A idade e atividade física moderada-vigorosa foram significativamente}

associados com MC na meia infância. O IMCz na meia infância e nas idade

de 4 e 5 anos explicou inversamente $12 \%$ ( $p<0,05), 6 \%(p>0,05)$ e $7 \%$ (p>

$0,05)$ da variação no CM em meninas após o ajuste para covariáveis, respectivamente. Em meninos não foram identificadas tendências.

Um programa de 16 semanas $\times 2 \mathrm{~h}$ de ginástica, demonstrou que as habilidades de estabilidade e controle de objetos mostraram um efeito significativo ( $p$ $<0,05)$ de interação intervenção x tempo. Nenhuma diferença foi encontrada nas habilidades locomotoras ou coordenação geral.

Houve correlação negativa e estatisticamente significativa entre o IMC e a coordenação motora $(r=-0,69 ; p<0,001)$. O grupo sobrepeso-obesidade apresentou menor desempenho no teste de coordenação motora comparado com o grupo eutrófico.

Crianças classificadas com sobrepeso/obesas tiveram um MQ menor do que CM diminui com o aumento do IMC em criancas.

As meninas que tinham menor peso, menor relação cintura-estatura e percentuais de gordura menos obtiveram melhores resultados nos testes de condicionamento físico. Melhores pontuações nos testes de condicionamento

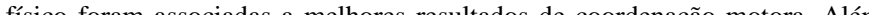
disso, a coodenac̃o a mella estava mis intimamente relacionala com a disso, a coordença motora estava mais in aptidão do que com as variáveis somáticas.

Os resultados mostraram que os pares irmã-irmã (SS) apresentaram maior semelhança no GMC $(\rho=0,24)$ em relação aos pares irmão-irmã (BS) ( $\rho=$ $0,10)$ e irmão-irmão (BB) $(\rho=0,07)$. Os pares BB tinham GMC mais alto do que os pares SS e os irmãos mais velhos tinham GMC mais alto do que os irmãos mais novos. Além disso, aqueles com menor IMC e maior PF tiveram maior GMC.

$\mathrm{O}$ rastreamento foi ruim para todos os testes de GMC $(0,17 \leq \kappa \leq 0,38)$ e moderado para o quociente motor de GMC (MQ) em meninos e meninas $(0,44$ $\leq \kappa \leq 0,45)$ A instabilidade nos extremos foi baixa em testes GMC insignificante para MQ. As crianças que apresentaram consistentemente n elevados de GMC apresentavam menor peso, menor IMC e gordura subcutânea e apresentaram pontuações mais altas em testes de aptidão física em comparação com criancas com GMC baixos.

Utilizou o teste KTK para avaliar coordenação Os resultados mostraram que a estrutura fatorial KTK foi adequada ao modelo

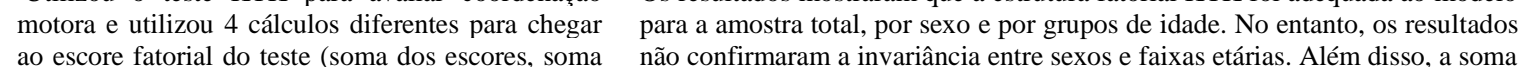


Research, Society and Development, v. 11, n. 2, e58111225955, 2022

(CC BY 4.0) | ISSN 2525-3409 | DOI: http://dx.doi.org/10.33448/rsd-v11i2.25955

\begin{tabular}{|c|c|c|c|c|}
\hline & $\begin{array}{l}\text { cálculo do escore fatorial do teste } \\
\text { (soma dos escores, soma dos } \\
\text { escores padrão, método ponderado } \\
\text { e método refinado). }\end{array}$ & & $\begin{array}{l}\text { dos escores padrão, método ponderado e método } \\
\text { refinado). }\end{array}$ & $\begin{array}{l}\text { das pontuações brutas dos subtestes poderia ser usada como o método de } \\
\text { pontuação do fator no KTK. }\end{array}$ \\
\hline $\begin{array}{l}\text { (Ochoa-Martínez } \\
\text { et al., 2019) }\end{array}$ & $\begin{array}{l}\text { Avaliar o efeito de um programa de } \\
\text { educação física adaptado em } \\
\text { escolares com deficiência auditiva } \\
\text { sobre o quociente motor. }\end{array}$ & $\begin{array}{l}38 \text { crianças (grupo } \\
\text { controle: } 11 \\
\text { meninos e } 4 \\
\text { meninas; grupo } \\
\text { experimental: } 15 \\
\text { meninos e } 8 \\
\text { meninas) } \\
\end{array}$ & $\begin{array}{l}\text { O programa durou quatro meses, cinco vezes por } \\
\text { semana, com duração de } 50 \text { minutos por sessão. O } \\
\text { quociente motor foi avaliado através do teste KTK. }\end{array}$ & $\begin{array}{l}\text { Um programa de atividade física adaptada durante } 4 \text { meses, } 5 \text { vezes por } \\
\text { semana, com duração de } 50 \text { minutos por sessão melhorou a coordenação } \\
\text { motora de crianças com deficiência auditiva. Ao comparar os resultados entre } \\
\text { os grupos da variável quociente motor, com a análise de variância (ANOVA) } \\
\text { mista } 2 \times 2 \text { foi observada uma interação duplamente significativa entre os } \\
\text { grupos e as medidas }(p=0,01) \text {, os percentuais de variação }(\Delta \%) \text { foram de } \\
26 \% \text { do grupo experimental e } 6,5 \% \text { do grupo controle }(\Delta \%) \text {. }\end{array}$ \\
\hline $\begin{array}{l}\text { (Popović et al., } \\
\text { 2020) }\end{array}$ & $\begin{array}{l}\text { Determinar as diferenças na } \\
\text { coordenação motora de crianças } \\
\text { matriculadas no futebol e em } \\
\text { atividades poliesportivas. }\end{array}$ & $\begin{array}{l}147 \text { ( } 88 \text { meninos e } \\
59 \text { meninas) }\end{array}$ & $\begin{array}{l}\text { A aptidão física foi avaliada com um teste de } 20 \mathrm{~m} \\
\text { teste de corrida em vaivém, } 4 \text { testes de corrida de } \\
\text { vaivém de } 10 \mathrm{~m} \text {, salto em distância em pé e força de } \\
\text { preensão manual. A coordenação motora foi } \\
\text { avaliada através do teste KTK. }\end{array}$ & $\begin{array}{l}\text { Os resultados demonstraram diferenças significativas }(\mathrm{p}<0,05) \text { com grande } \\
\text { tamanho de efeito entre os grupos para testes de salto para altura }(\mathrm{d}=0,93) \text {, } \\
\text { quociente motor total }(\mathrm{d}=1,31) \text {, salto lateral }(\mathrm{d}=1,32) \text { e movimento lateral }(\mathrm{d} \\
=1,59) \text {. Crianças matriculadas em atividades poliesportivas possuem níveis } \\
\text { mais elevados de coordenação motora do que crianças matriculadas apenas no } \\
\text { futebol. }\end{array}$ \\
\hline $\begin{array}{l}\text { (Niemistö et al., } \\
\text { 2020) }\end{array}$ & $\begin{array}{l}\text { Examinar a competência motora } \\
\text { (CM) através de duas ferramentas } \\
\text { diferentes em relação ao indivíduo, } \\
\text { família e correlatos ambientais e } \\
\text { investigar diferenças de gênero em } \\
\text { CM. }\end{array}$ & $\begin{array}{l}945 \text { crianças }(473 \\
\text { meninos e } 472 \\
\text { meninas) }\end{array}$ & $\begin{array}{l}\text { A avaliação da coordenação motora foi avaliada } \\
\text { através do Test of Gross Motor Development-Third } \\
\text { Edition (TGMD-3) e teste KTK. Foi utilizado um } \\
\text { questionário para saber sobre costumes individuais. } \\
\text { O temperamento das crianças foi avaliado pelo } \\
\text { questionário Colorado Childhood Temperament } \\
\text { Inventory (CCTI). }\end{array}$ & $\begin{array}{l}\text { Os correlatos individuais, incluindo idade avançada, participação mais } \\
\text { frequente em esportes e características de temperamento específicas de } \\
\text { atividade e atenção-persistência, foram os preditores mais fortes para um } \\
\text { melhor MC. Houve uma pequena diferença entre os gêneros em ambos os } \\
\text { instrumentos. }\end{array}$ \\
\hline $\begin{array}{l}\text { (V. P. Lopes et } \\
\text { al., 2020) }\end{array}$ & $\begin{array}{l}\text { Identificar classes de diferentes } \\
\text { trajetórias de desenvolvimento do } \\
\text { IMC e testá-las para diferenças na } \\
\text { competência motora (CM) e aptidão } \\
\text { cardiorrespiratória (CFR), em } \\
\text { crianças e adolescentes }\end{array}$ & $\begin{array}{l}147 \text { crianças }(78 \\
\text { meninos e } 69 \\
\text { meninas) }\end{array}$ & $\begin{array}{l}\text { Foram medidos dados antropométricos (altura, peso, } \\
\text { índice de massa corporal (IMC)). O CM foi } \\
\text { avaliado através do teste KTK e TGMD-2. O CRF } \\
\text { foi avaliado através de uma corrida/caminhada de } \\
\text { uma milha. }\end{array}$ & $\begin{array}{l}\text { Duas classes significativas foram identificadas. As crianças pertencentes a } \\
\text { classe } 1 \text { ( } 78.92 \% \text { dos participantes) apresentaram IMC inicial inferior e } \\
\text { declive inferior em relaçãa à classe } 2 \text { ( } 21,08 \% \text { dos participantes). As crianças } \\
\text { da classe } 1 \text { tiveram melhores resultados de coordenação motora. }\end{array}$ \\
\hline $\begin{array}{l}\text { (Barros et al., } \\
2020)\end{array}$ & $\begin{array}{l}\text { Analisar os efeitos do treinamento } \\
\text { de canoagem durante oito semanas } \\
\text { sobre as habilidades motoras } \\
\text { grossas em crianças e adolescentes } \\
\text { com síndrome de Down. }\end{array}$ & $\begin{array}{l}7 \text { crianças (4 } \\
\text { meninos e } 3 \\
\text { meninas) }\end{array}$ & $\begin{array}{l}\text { O teste KTK foi utilizado para avaliar a } \\
\text { coordenação motora. }\end{array}$ & $\begin{array}{l}\text { Um programa de treinamento de } 16 \text { semanas em canoagem apresentou } \\
\text { resultados estatisticamente significativos em todas as tarefas do teste de } \\
\text { coordenação motora grossa e o efeito produzido pela intervenção foi } \\
\text { moderado. }\end{array}$ \\
\hline $\begin{array}{l}\text { (Almeida et al., } \\
\text { 2021) }\end{array}$ & $\begin{array}{l}\text { Examinar os efeitos do treinamento } \\
\text { pliométrico na aptidão física (FP) e } \\
\text { na coordenação motora grossa } \\
\text { (GMC) de escolares de } 7 \text { a } 9 \text { anos. }\end{array}$ & $\begin{array}{l}116 \text { meninos } \\
\text { (grupo de } \\
\text { treinamento } \\
\text { pliométrico (PT, } \mathrm{n} \\
=73) \text { e grupo de } \\
\text { controle }(\mathrm{GC}, \mathrm{n}= \\
\text { 43)). } \\
\end{array}$ & $\begin{array}{l}\text { Foram avaliados o índice de massa corporal (IMC), } \\
\text { coordenação motora grossa através do teste KTK, } \\
\text { força de preensão manual, salto em distância em pé } \\
\text { (SLJ), flexões, sentar e alcançar, quadrado-teste, } \\
\text { velocidade de corrida e teste de corrida de } 1 \text { milha. } \\
\text { O treinamento consistia em exercícios } 2 \text { X semana } \\
\text { em dias não consecutivos por } 12 \text { semanas. }\end{array}$ & $\begin{array}{l}\text { O treinamento pliométrico ( } 12 \text { semanas, duas vezes/semana, } 20 \mathrm{~min} / \mathrm{dia}) \\
\text { melhorou os componentes de coordenação motora grossa e aptidão física. Os } \\
\text { resultados do grupo treinamento foram lehores que o do grupo controle no } \\
\text { teste de flexibilidade }(\mathrm{GC}=-2,7 \% \text { e } \mathrm{PT}=3,5 \%) \text {, salto lateral }(\mathrm{GC}=16,3 \% \text { e } \\
\mathrm{PT}=17,4 \% \text { e pontuação geral do } \mathrm{GMC}(\mathrm{GC}=9,8 \% \text { e } \mathrm{PT}=15,9 \%) .\end{array}$ \\
\hline
\end{tabular}

Fonte: Autores. 


\section{Discussão}

O objetivo deste estudo foi identificar o que tem sido publicado a respeito da utilização do teste KTK nos últimos anos para avaliação da coordenação motora de crianças, e secundariamente analisar a relação entre coordenação motora, antropometria e desempenho. Dessa maneira, os principais resultados acerca disto foram: a) medidas antropométricas apresentam uma relação inversamente proporcional com a coordenação motora; b) o desempenho apresenta uma relação diretamente proporcional e C) fatores antropométricos, idade avançada, prática esportiva e características de temperamento específicas de atividade e atenção-persistência, são os preditores mais fortes para uma melhor coordenação motora.

As medidas antropométricas de peso, percentual de gordura, índice de massa corporal, circunferência da cintura, a razão cintura/estatura e estatura, são inversamente proporcionais à coordenação motora, dessa forma crianças que apresentam valores elevados nesses parâmetros apresentam uma pior coordenação motora grossa quando comparados com crianças que apresentam valores normais (Freitas et al., 2017; Lopes et al., 2018, 2020; Luz et al., 2016). Entretanto, crianças que nasceram com baixo peso, também apresentam resultados piores em comparação com crianças que nasceram com peso normal (Tchamo et al., 2017). Esses resultados estão de acordo com o encontrado em uma metanálise que associou o IMC com o desempenho no teste KTK. Nesta metanálise, os autores encontraram como resultados que valores maiores de IMC estão diretamente associados ao baixo desempenho no KTK e que a magnitude do efeito de associação foi classificada como pequena, porém, significativa para o sexo masculino ( $\mathrm{r}=0,29 ; \mathrm{IC} 95 \% 0,27$ a 0,$32 ; \mathrm{Z}=22,47 ; \mathrm{p}=0,000)$ e moderada para o sexo feminino $(\mathrm{r}=0,32$; IC95\% 0,30 a 0,34; $Z=24,76 ; p=0,000$ ) ( Luz et al., 2015). Dentro deste contexto, o estudo de Freitas et al. (2017) demonstrou que atletas com um IMC mais elevado apresentaram menores valores no teste KTK, porém para os atletas considerados eutróficos não houve uma associação positiva entre IMC e os resultados de coordenação motora grossa. Dessa forma o treinamento não parece ser capaz de igualar os resultados motores de jovens atletas com sobrepeso com o de crianças com peso normal (Freitas et al., 2017).

Nessa perspectiva, o sobrepeso em crianças está associado com um maior tempo sedentário e consequentemente menor inserção em práticas esportivas, o que afeta negativamente a coordenação motora (Han et al., 2018) e crianças com distúrbios na coordenação motora tendem a não se envolverem em atividades físicas em decorrência do medo de falharem, criando assim um ciclo vicioso (Hendrix et al., 2014). Nesse sentido, um estudo realizado com 668 crianças, identificou que o IMC elevado pode afetar negativamente a coordenação motora, porém o inverso não é verdadeiro, ou seja, uma pior coordenação não afeta negativamente o IMC de uma criança (Cheng et al., 2016). O efeito do sobrepeso/obesidade sobre a coordenação motora pode ser explicada por meio da biomecânica, o aumento do peso leva à uma alteração da geometria corporal, alterando o centro de massa do corpo (AlAbdulwahab \& Kachanathu, 2016) e aumentando o peso dos segmentos corporais (D’Hondt et al., 2011), levando a movimentos biomecânicamente ineficientes.

Além da composição corporal, como dito anteriormente, a prática regular de atividades físicas apresenta influência clara nos resultados do teste KTK (Nascimento et al., 2019). A prática regular de atividades físicas promove a manutenção da força e elasticidade muscular normais, o que possibilita a realização coordenada dos movimentos. A relação entre atividade física e coordenação motora varia ao longo do amadurecimento biológico da criança. Em crianças mais jovens, a atividade física é um importante meio pelo qual a criança irá desenvolver habilidades motoras, já em crianças mais velhas esse papel se inverte, sendo as habilidades motoras necessárias para a participação em atividades físicas específicas (Stodden et al., 2008). Além disso, diferentes atividades irão desenvolver diferentes aspectos que podem contribuir para uma melhor coordenação motora (Almeida et al., 2021; Nobre et al., 2017; Ochoa-Martínez et al., 2019; Rudd et al., 2017). A maioria dos estudos incluídos aqui, que investigaram o efeito de um determinado treinamento sobre a coordenação motora, utilizaram exercícios pliométricos e encontraram resultados positivos através dessas intervenções. 
O KTK é um teste confiável e de baixo custo, utilizado para avaliar a coordenação motora de crianças, sendo utilizado em vários países (Bardid et al., 2015; Cattuzzo et al., 2016). No estudo de Santos et al. (2020), os autores avaliaram a estrutura fatorial do teste KTK em crianças em idade escolar e confirmaram que o teste possui capacidade de investigar e classificar o nível de coordenação motora de crianças, identificando possíveis alterações nesse aspecto (J. O. L. dos Santos et al., 2020).

\section{Conclusão}

O KTK é um teste confiável para avaliar a coordenação motora grossa de crianças. A literatura recente demonstra que esse teste possui relações inversamente proporcionais e diretamente proporcionais com medidas antropométricas e o desempenho, respectivamente.

\section{Referências}

AlAbdulwahab, S. S., \& Kachanathu, S. J. (2016). Effects of body mass index on foot posture alignment and core stability in a healthy adult population. Journal of Exercise Rehabilitation, 12(3), 182-187. https://doi.org/10.12965/jer.1632600.300

Almeida, M. B. de, Leandro, C. G., Queiroz, D. da R., José-da-Silva, M., Pessôa Dos Prazeres, T. M., Pereira, G. M., das-Neves, G. S., Carneiro, R. C., Figueredo-Alves, A. D., Nakamura, F. Y., Henrique, R. D. S., \& Moura-Dos-Santos, M. A. (2021). Plyometric training increases gross motor coordination and associated components of physical fitness in children. European Journal of Sport Science, 21(9), 1263-1272. https://doi.org/10.1080/17461391.2020.1838620

Bardid, F., Rudd, J., Lenoir, M., Polman, R., \& Barnett, L. (2015). Cross-cultural comparison of motor competence in children from Australia and Belgium. Frontiers in Psychology, 6, 964. https://doi.org/10.3389/fpsyg.2015.00964

Barros, N. P., Araújo, D. de S., Lima, E. B. de, Carvalho, I. P. de, Lima, S. da S., \& Costa, L. G. T. (2020). Effects of kayaking on motor skills in children and adolescents with Down Syndrome. Revista Brasileira de Medicina Do Esporte, 26, 302-306. https://doi.org/10.1590/1517-869220202604220312

Bernshtein, N. A. (1967). The co-ordination and regulation of movements. Pergamon Press. http://books.google.com/books?id=F9dqAAAAMAAJ

Buratti, J. R., Souza, N. C., \& Gorla, J. I. (2020). Coordenação motora. In Portal de Livros de Acesso Aberto (Brasil; Contemporâneo). Portal de Livros de Acesso Aberto. https://doi.org/10.20396/ISBN9786588397077

Cattuzzo, M. T., dos Santos Henrique, R., Ré, A. H. N., de Oliveira, I. S., Melo, B. M., de Sousa Moura, M., de Araújo, R. C., \& Stodden, D. (2016). Motor competence and health related physical fitness in youth: A systematic review. Journal of Science and Medicine in Sport, 19(2), 123-129. https://doi.org/10.1016/j.jsams.2014.12.004

Cheng, J., East, P., Blanco, E., Sim, E. K., Castillo, M., Lozoff, B., \& Gahagan, S. (2016). Obesity Leads to Declines in Motor Skills across Childhood. Child: Care, Health and Development, 42(3), 343. https://doi.org/10.1111/cch.12336

D’Hondt, E., Deforche, B., Vaeyens, R., Vandorpe, B., Vandendriessche, J., Pion, J., Philippaerts, R., de Bourdeaudhuij, I., \& Lenoir, M. (2011). Gross motor coordination in relation to weight status and age in 5- to 12-year-old boys and girls: A cross-sectional study. International Journal of Pediatric Obesity: IJPO: An Official Journal of the International Association for the Study of Obesity, 6(2-2), e556-564. https://doi.org/10.3109/17477166.2010.500388

Freitas, J. V. de, Castro, P. H. C. de, Rezende, E. C., Werneck, F. Z., \& Lima, J. R. P. de. (2017). Relação entre o excesso de peso e a coordenação motora de jovens atletas de atletismo. Revista Brasileira de Ciências do Esporte, 39, 91-97. https://doi.org/10.1016/j.rbce.2016.02.003

Gorla, J. I., Araujo, P. F., \& Rodrigues, J. L. (2009). Avaliação Motora Em Educação Fisica Adaptada Teste KTK (2a ed.). Phorte.

Han, A., Fu, A., Cobley, S., \& Sanders, R. H. (2018). Effectiveness of exercise intervention on improving fundamental movement skills and motor coordination in overweight/obese children and adolescents: A systematic review. Journal of Science and Medicine in Sport, 21(1), 89-102. https://doi.org/10.1016/j.jsams.2017.07.001

Hendrix, C. G., Prins, M. R., \& Dekkers, H. (2014). Developmental coordination disorder and overweight and obesity in children: A systematic review. Obesity Reviews: An Official Journal of the International Association for the Study of Obesity, 15(5), 408-423. https://doi.org/10.1111/obr.12137

Henrique, R. S., Bustamante, A. V., Freitas, D. L., Tani, G., Katzmarzyk, P. T., \& Maia, J. A. (2018). Tracking of gross motor coordination in Portuguese children. Journal of Sports Sciences, 36(2), 220-228. https://doi.org/10.1080/02640414.2017.1297534

Laukkanen, A., Pesola, A. J., Finni, T., \& Sääkslahti, A. (2017). Body Mass Index in the Early Years in Relation to Motor Coordination at the Age of 57 Years. Sports (Basel, Switzerland), 5(3), E49. https://doi.org/10.3390/sports5030049

Lima, R. A., Bugge, A., Pfeiffer, K. A., \& Andersen, L. B. (2017). Tracking of Gross Motor Coordination From Childhood Into Adolescence. Research Quarterly for Exercise and Sport, 88(1), 52-59. https://doi.org/10.1080/02701367.2016.1264566

Lopes, L., Póvoas, S., Mota, J., Okely, A. D., Coelho-E-Silva, M. J., Cliff, D. P., Lopes, V. P., \& Santos, R. (2017). Flexibility is associated with motor competence in schoolchildren. Scandinavian Journal of Medicine \& Science in Sports, 27(12), 1806-1813. https://doi.org/10.1111/sms.12789 
Lopes, L., Santos, R., Moreira, C., Pereira, B., \& Lopes, V. P. (2015). Sensitivity and specificity of different measures of adiposity to distinguish between low/high motor coordination. Jornal de Pediatria, 91, 44-51. https://doi.org/10.1016/j.jped.2014.05.005

Lopes, V. P., Malina, R. M., Maia, J. a. R., \& Rodrigues, L. P. (2018). Body mass index and motor coordination: Non-linear relationships in children 6-10 years. Child: Care, Health and Development, 44(3), 443-451. https://doi.org/10.1111/cch.12557

Lopes, V. P., Utesch, T., \& Rodrigues, L. P. (2020). Classes of developmental trajectories of body mass index: Differences in motor competence and cardiorespiratory fitness. Journal of Sports Sciences, 38(6), 619-625. https://doi.org/10.1080/02640414.2020.1722024

Luz, L. G. de O., Teixeira e Seabra, A. F., Santos, R., Padez, C., Ferreira, J. P., \& Coelho-e-Silva, M. J. (2015). Associação entre IMC e teste de coordenação corporal para crianças (KTK). Uma meta-análise. Revista Brasileira de Medicina do Esporte, 21, 230-235. https://doi.org/10.1590/1517869220152103144469

Luz, L. G. O., Coelho-e-Silva, M. J., Duarte, J. P., Valente-dos-Santos, J., Machado-Rodrigues, A., Seabra, A., Carmo, B. C. M., Vaeyens, R., Philippaerts, R. M., Cumming, S. P., \& Malina, R. M. (2018). Multivariate Relationships among Morphology, Fitness and Motor Coordination in Prepubertal Girls. Journal of Sports Science \& Medicine, 17(2), 197.

Luz, L. G. O., Seabra, A., Padez, C., Duarte, J. P., Rebelo-Gonçalves, R., Valente-dos-Santos, J., Luz, T. D. D., Carmo, B. C. M., \& Coelho-e-Silva, M. (2016). Waist circumference as a mediator of biological maturation effect on the motor coordination in children. Revista Paulista de Pediatria, 34, 352-358. https://doi.org/10.1016/j.rppede.2016.02.004

Moher, D., Liberati, A., Tetzlaff, J., \& Altman, D. G. (2009). Preferred Reporting Items for Systematic Reviews and Meta-Analyses: The PRISMA Statement. Physical Therapy. https://doi.org/10.1093/ptj/89.9.873

Moreira, J. P. A., Lopes, M. C., Miranda-Júnior, M. V., Valentini, N. C., Lage, G. M., \& Albuquerque, M. R. (2019). Körperkoordinationstest Für Kinder (KTK) for Brazilian Children and Adolescents: Factor Analysis, Invariance and Factor Score. Frontiers in Psychology, 10, 2524. https://doi.org/10.3389/fpsyg.2019.02524

Nascimento, W. M. do, Henrique, N. R., \& Marques, M. da S. (2019). KTK MOTOR TEST: REVIEW OF THE MAIN INFLUENCING VARIABLES. Revista Paulista de Pediatria, 37(3), 372-381. https://doi.org/10.1590/1984-0462/;2019;37;3;00013

Niemistö, D., Finni, T., Cantell, M., Korhonen, E., \& Sääkslahti, A. (2020). Individual, Family, and Environmental Correlates of Motor Competence in Young Children: Regression Model Analysis of Data Obtained from Two Motor Tests. International Journal of Environmental Research and Public Health, 17(7), E2548. https://doi.org/10.3390/ijerph17072548

Nobre, G. G., de Almeida, M. B., Nobre, I. G., Dos Santos, F. K., Brinco, R. A., Arruda-Lima, T. R., de-Vasconcelos, K. L., de-Lima, J. G., Borba-Neto, M E., Damasceno-Rodrigues, E. M., Santos-Silva, S. M., Leandro, C. G., \& Moura-Dos-Santos, M. A. (2017). Twelve Weeks of Plyometric Training Improves Motor Performance of 7- to 9-Year-Old Boys Who Were Overweight/Obese: A Randomized Controlled Intervention. Journal of Strength and Conditioning Research, 31(8), 2091-2099. https://doi.org/10.1519/JSC.0000000000001684

Ochoa-Martínez, P. Y., Hall-López, J. A., Carmona López, A. A., Morales Ramírez, M. M., Alarcón Meza, E. I., Sáenz-López Buñuel, P., Ochoa-Martínez, P. Y., Hall-López, J. A., Carmona López, A. A., Morales Ramírez, M. M., Alarcón Meza, E. I., \& Sáenz-López Buñuel, P. (2019). Efecto de un programa adaptado de educación física en niños con discapacidad auditiva sobre la coordinación motora. MHSalud, 16(2), 17-28. https://doi.org/10.15359/mhs.16-2.2

Popović, B., Gušić, M., Radanović, D., Andrašić, S., Madić, D. M., Mačak, D., Stupar, D., Đukić, G., Grujičić, D., \& Trajković, N. (2020). Evaluation of Gross Motor Coordination and Physical Fitness in Children: Comparison between Soccer and Multisport Activities. International Journal of Environmental Research and Public Health, 17(16), E5902. https://doi.org/10.3390/ijerph17165902

Rudd, J. R., Barnett, L. M., Farrow, D., Berry, J., Borkoles, E., \& Polman, R. (2017). Effectiveness of a 16 week gymnastics curriculum at developing movement competence in children. Journal of Science and Medicine in Sport, 20(2), 164-169. https://doi.org/10.1016/j.jsams.2016.06.013

Santos, A. (2002). A biomecânica da coordenação motora ( $3^{\text {a }}$ edição). Summus Editorial.

Santos, J. O. L. dos, Medeiros, P. de, Cardoso, F. L., Formiga, N. S., Souza, N. C., \& Gorla, J. I. (2020). Validação da estrutura fatorial do Körperkoordination Test für Kinder (KTK) em escolares de 8 a 10 anos. Saúde e Desenvolvimento Humano, 8(3), 31-37. https://doi.org/10.18316/sdh.v8i3.6060

Söğüt, M. (2016). Gross motor coordination in junior tennis players. Journal of Sports Sciences, 34(22), 2149-2152. https://doi.org/10.1080/02640414.2016.1211311

Stodden, D. F., Goodway, J. D., Langendorfer, S. J., Roberton, M. A., Rudisill, M. E., Garcia, C., \& Garcia, L. E. (2008). A Developmental Perspective on the Role of Motor Skill Competence in Physical Activity: An Emergent Relationship. Quest, 60(2), 290-306. https://doi.org/10.1080/00336297.2008.10483582

Tchamo, M. E., Moura-Dos-Santos, M. A., Dos Santos, F. K., Prista, A., \& Leandro, C. G. (2017). Deficits in anthropometric indices of nutritional status and motor performance among low birth weight children from Maputo City, Mozambique. American Journal of Human Biology: The Official Journal of the Human Biology Council, 29(3). https://doi.org/10.1002/ajhb.22949

Valdívia, A. B., Henrique, R. S., Pereira, S., Chaves, R. N., Tani, G., Freitas, D., Prista, A., Stodden, D. F., Katzmarzyk, P. T., Hedeker, D., \& Maia, J. (2018). Familial resemblance in gross motor coordination. The Peruvian Sibling Study on Growth and Health. Annals of Human Biology, 45(6-8), 463-469. https://doi.org/10.1080/03014460.2019.1568549. 